ORIGINAL ARTICLE

\title{
Detection of chest pain of non-cardiac origin at the emergency room by a new non-invasive device avoiding unnecessary admission to hospital
}

\author{
M Gorenberg, A Marmor, H Rotstein
}

Emerg Med J 2005;22:486-489. doi: 10.1136/emj.2004.016188

Recent advances in the treatment of acute coronary syndromes has raised awareness that prompt presentation for chest pain may be life saving. Most patients presenting with chest discomfort have a nonischaemic ECG on presentation, but are routinely admitted to hospital because of diagnostic uncertainty for occult Ml or ischaemia.

We tested a new non-invasive device that measures central aortic pressure changes $\left(\mathrm{dP} / \mathrm{dt}_{\text {ejc }}\right)$ : an accepted index of myocardial performance that could be added to the diagnostic triage of ischaemia in the ER avoiding unnecessary admissions.

See end of article for authors' affiliations We followed 85 patients presenting at the ER with acute chest pain. In 72 patients, negative ECG and myocardial enzyme dynamics ruled out coronary origin during the first $24 \mathrm{~h}$ after admission. In 8 of the 72 patients, coronary catheterisation found normal coronary arteries. In this group, average $\mathrm{dP} / \mathrm{dt}_{\text {ejc }}$ was 163 (range 92-232). In 35 patients in whom the new non-invasive cardiac performance index $\mathrm{dP} / \mathrm{dt}_{\text {ejc }}$ was above a threshold of $>150$, acute $\mathrm{Ml}$ was ruled out. In 13 patients, acute chest pain had coronary origin confirmed by ECG and/or positive enzymes. The average $\mathrm{dP} / \mathrm{dt}_{\text {ejc }}$ in this group was 117 (range 61-149). The $\mathrm{dP} / \mathrm{dt}_{\text {ejc }}$ values were found to be significantly higher in patients without acute $\mathrm{Ml}(\mathrm{p}<0.001)$.

Preliminary findings suggest that nearly $40 \%$ of patients presenting with acute chest pain could be spared the risks and costs of unnecessary hospital admission and more invasive cardiac testing by simply adding a easy to use, immediately obtained, test to the diagnostic protocol, and using a threshold of $\mathrm{dP} / \mathrm{dt}_{\mathrm{ej}}$ $>150$ to rule out heart attack.

Accepted for publication 28 July 2004

$\mathrm{T}$ he decision to discharge patients with chest pain from non-cardiac origin from the emergency room (ER) is challenging. There are many uncertainties and legal aspects, which makes the decision difficult. One of the problems with chest pain patients presenting to ER is that diagnosis is deferred for at least $6 \mathrm{~h}$ when the enzymatic markers become diagnostic. An early immediate diagnosis of chest pain of non-cardiac origin may be of great help for the clinician to avoid unnecessary admissions.

It has been shown that during acute myocardial ischaemia, cardiac contractility decreases. One of the most sensitive indexes of contractility is the left ventricular $\mathrm{dP} / \mathrm{dt}$ and arterial $\mathrm{dP} / \mathrm{dt}$, which represents the rate of increase of intraventricular pressure during isovolumetric contraction; ejection $\mathrm{dP} / \mathrm{dt}$ represents the rate of change of pressure during ejection that has been shown to be also sensitive to changes in contractility. ${ }^{1-4}$ We hypothesise that chest pain of non-cardiac origin will generate a higher value of $\mathrm{dP} / \mathrm{dt}$ than ischaemic chest pain because ischaemia reduces myocardial performance whereas chest pain of non-cardiac origin increases $\mathrm{dP} / \mathrm{dt}$ by the sheer stress of pain. We tested a new non-invasive device that measures central aortic pressure changes $\left(\mathrm{dP} / \mathrm{dt}_{\mathrm{ejc}}\right)$, which could be added to the diagnostic triage of ischaemia in the ER avoiding unnecessary admissions.

\section{METHODS}

\section{Patient population and setting}

The study was performed in a university affiliated teaching hospital emergency department with 30000 visits annually. The study group consisted of 85 patients admitted to the ER for chest pain. We included patients if they were aged over 18 years, the chest pain had lasted less than $12 \mathrm{~h}$, there was no history of trauma, and no other medical causes of chest pain had been diagnosed. We excluded patients if they had arrhythmia, or if the protocol was not followed, or they did not consent to participate. The study complies with the Declaration of Helsinki. The locally appointed ethics committee approved the research protocol and informed consent was obtained from all of the patients.

\section{Study protocol}

All patients had three consecutive measurements of $\mathrm{dP} / \mathrm{dt}_{\text {ejc }}$ and the average value was calculated. The best threshold value of $\mathrm{dP} / \mathrm{dt}_{\mathrm{ejc}}>150$ to rule out acute myocardial infarction (MI) was found using ROC analysis. ${ }^{5}$ All patients with a value above the threshold were considered to have chest pain of non-ischaemic origin. In order to prove our hypothesis all patients were admitted to hospital and an acute coronary event was ruled out by repeated 12 lead electrocardiography (ECG) and repeated measurements of CPK-MB and troponin over the first $24 \mathrm{~h}$ after admission. The group comprised $6 \mathrm{l}$ men and 24 women; mean age 62 years (range 19-95).

\section{Measurements}

The device

The ascending limb of the aortic pressure $\left(\mathrm{dP} / \mathrm{dt}_{\mathrm{ejc}}\right)$ was measured non-invasively with a newly designed computer controlled device (CardioWatch Ltd., Matam Advanced Technology Center, Haifa, Israel) (fig 1).

Abbreviations: ECG, electrocardiogram; ER, emergency room; MI, myocardial infarction 


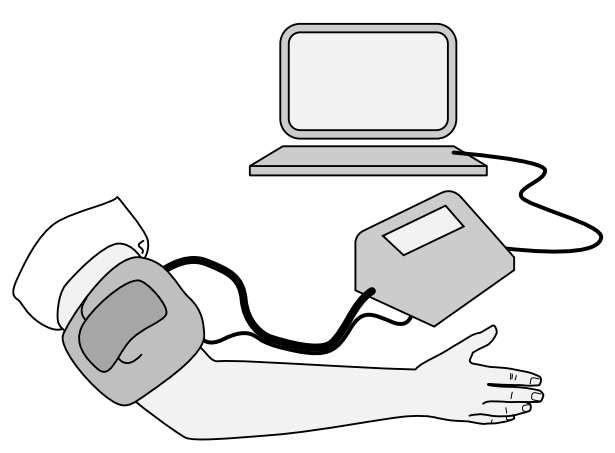

Figure 1 The device consists of three components: (1) a sphyngomonometric arm cuff attached to an air pressure unit; (2) an array of proprietary sensors attached to the arm at the antecubital space over the brachial artery, and (3) a computerised monitoring system.

This device consists of three components: (1) a sphygmonometric arm cuff attached to an air pressure unit, (2) an array of proprietary sensors attached to the arm at the antecubital space over the brachial artery (fig 2), and (3) a computerised monitoring system.

By applying occlusive pressure over the brachial artery during systole using non-inflatable cuff, a temporary standing fluid column is created in which the rising intra-aortic pressure is transmitted to the periphery with minimal distortion. This standing column of blood may be regarded as though it were a manometric tube installed directly into the aortic arch.

The time intervals required for the aortic pressure wave to overcome a given occlusive brachial pressure applied by a sphyngomanometer on the arm are equal to time needed to reach the same pressure in the central aorta plus the propagation time to the brachial point, which is constant in the same patient throughout the measurements. Time intervals are measured from the onset of flow breakthrough at the first sensor (fig 3) to the breakthrough reached at succeeding sensors.

Applying multiple successive occlusive pressures on the brachial artery from peak systole to diastole and plotting the values against time intervals described above results in the reconstruction of the central aortic pressure curve. From the ascending arterial pressure wave form, we can calculate the $\mathrm{dP} / \mathrm{dt}_{\mathrm{ejc}}$ - the index used in the present study. Noninvasive systolic pressure wave forms are generated by measuring time delay between the first sensor on the array serving as a reference sensor and the onset of brachial artery flow in the subsequent sensors during controlled upper arm deflation. The delay decreases with falling cuff pressure so that the plot of pressure versus time delays yields the

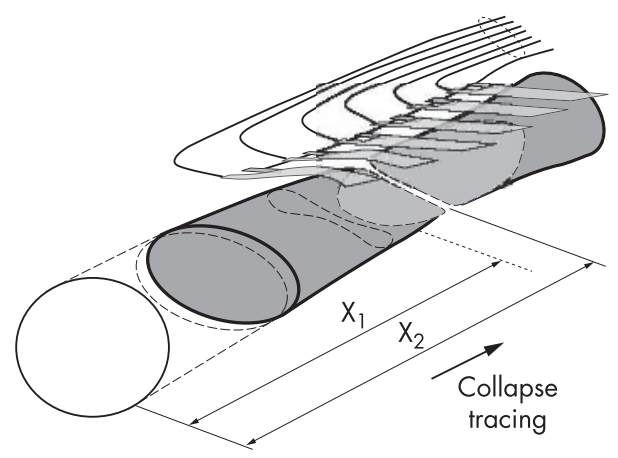

Figure 2 Detailed view of the new sensor system and artery with the collapsed region under the cuff. Upstream pressure is systolic.

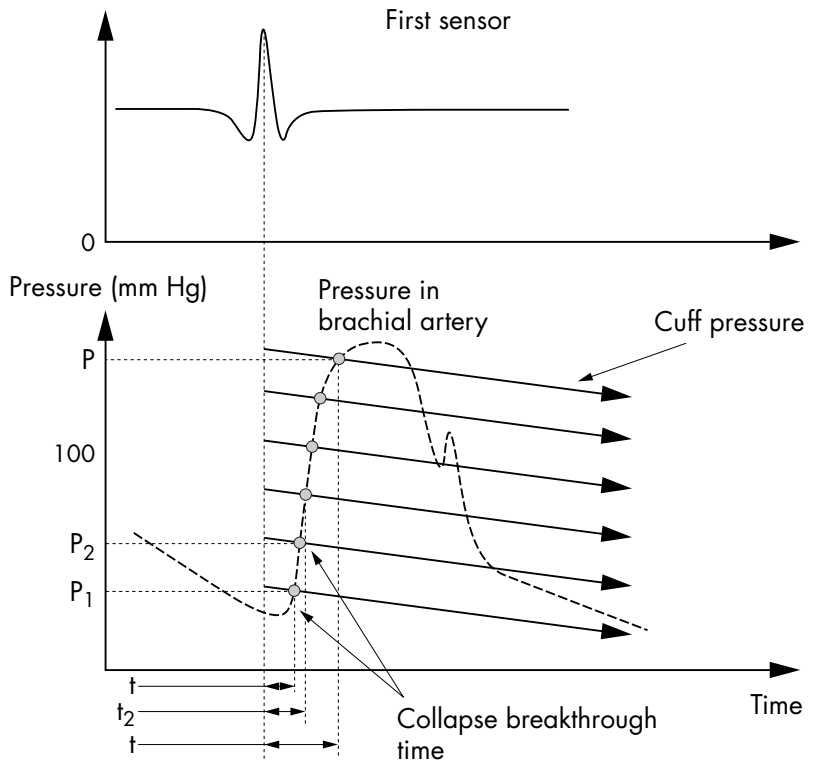

Figure 3 Measurements of the time delay between the first sensor on the array serving as a reference sensor and the onset of brachial artery flow in the subsequent sensors during controlled upper arm deflation. The plot of pressure $v$ time delays yields the ascending portion of the arterial wave form.

ascending portion of the arterial wave form (fig 4). Previously, ECG was used as a reference point instead of the first sensor; ${ }^{6-8}$ however, because the delay between onset of $\mathrm{R}$ wave on ECG and the first sensor on the arm is constant $(130 \pm 17 \mathrm{msec})$, we can use the first sensor instead of ECG. ${ }^{9}$ The pressure wave thus generated is identical to the pressure wave generated using the ECG reference system with constant delay. These wave forms were validated previously with simultaneously obtained invasive ascending aortic pressure and a correlation coefficient of $\mathrm{r}=0.98$ was found. ${ }^{\circ}$

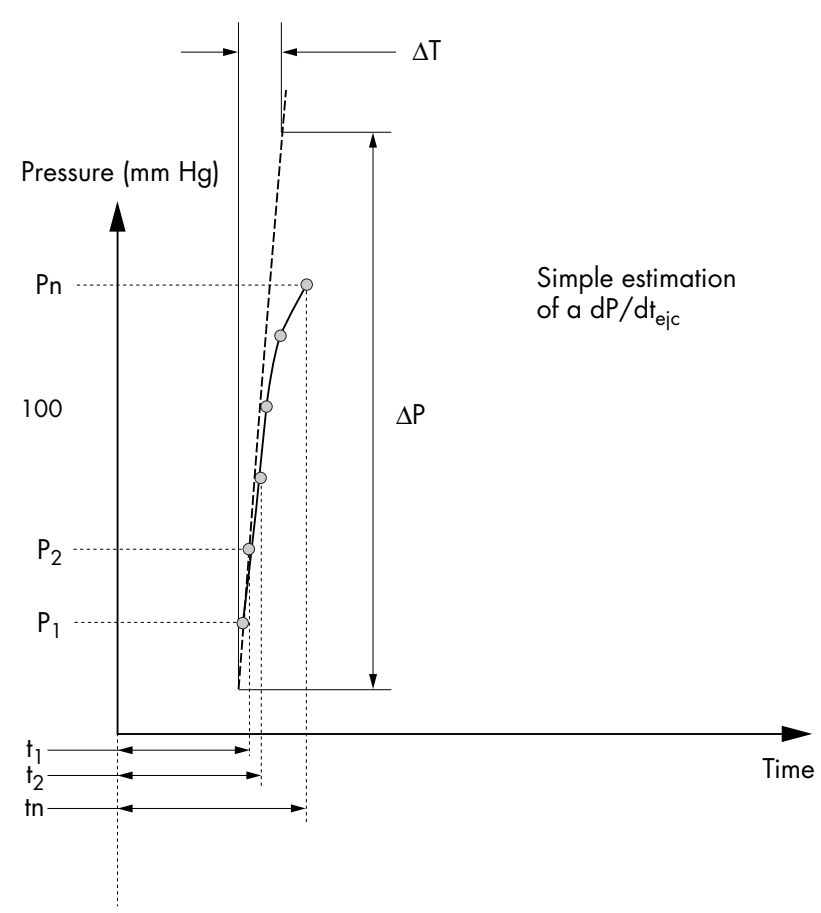

Figure 4 Reconstruction of a pressure wave form in brachial artery. 


\section{Reproducibility}

The reproducibility of measurements 2 minutes apart prior to initiation of catheterisation was measured in 63 patients (46 men and 17 women, mean age $65 \pm 13$ years) who subsequently underwent angioplasty. The difference in the measurements was $0.2 \pm 5.8$. $^{\circ}$

\section{Statistical analysis}

Data were expressed as mean \pm standard deviation. Comparisons between groups were performed with Student's unpaired $t$ test and Wilcoxon unpaired simple test. Statistical significance was achieved at the $5 \%$ level $(p<0.05)$. ROC analysis was used to establish the best threshold value for $\mathrm{dP} / \mathrm{dt}_{\mathrm{ejc} .}{ }^{5}$

\section{RESULTS}

We followed 85 patients presenting at the ER with acute chest pain (fig 5). In 72 patients, coronary origin was ruled out by negative ECG and myocardial enzyme dynamics during the first $24 \mathrm{~h}$ after admission. In 8 of the 72 patients, coronary catheterisation found normal coronary arteries. In this group, average $\mathrm{dP} / \mathrm{dt}_{\mathrm{ejc}}$ was 163 (range 92-232). In 35 patients in whom our new non-invasive cardiac performance index $\mathrm{dP} / \mathrm{dt}_{\text {ejc }}$ was above a threshold of $>150$, acute MI was ruled out. In 13 patients, acute chest pain had coronary origin confirmed by ECG and/or positive enzymes. The average $\mathrm{dP} /$ $\mathrm{dt}_{\mathrm{ejc}}$ in this group was 117 (range 61-149). The $\mathrm{dP} / \mathrm{dt}_{\mathrm{ejc}}$ values were found to be significantly higher in patients without acute MI $(p<0.001)$. The following criteria were applied: true positive results were considered when patients show positive enzymes dynamics and/or ECG changes compatible with ischaemia and $\mathrm{dP} / \mathrm{dt}_{\mathrm{ejc}} \leqslant 150$; true negative results when patients show negative enzymes dynamics and normal ECG and $\mathrm{dP} / \mathrm{dt}_{\mathrm{ejc}} \geqslant 150$; false positive results when patients show negative enzymes dynamics and normal ECG and $\mathrm{dP} /$ $\mathrm{dt}_{\text {ejc }} \leqslant 150$; false negative results when patients show positive enzymes dynamics and/or ECG changes compatible with ischaemia and $\mathrm{dP} / \mathrm{dt}_{\mathrm{ejc}} \geqslant 150$.

By ROC analysis, a threshold of 150 was found to be the best with a sensitivity of $100 \%$, negative predictive value $100 \%$, specificity $61 \%$, positive predictive value $32 \%$, and accuracy $67 \%$.

All patients with $\mathrm{dP} / \mathrm{dt}_{\mathrm{ejc}} \geqslant 150$ were discharged after at least 1 day of admission and MI was not identified in any patient. Using this criteria, nearly $40 \%$ of patients presenting with acute chest pain could be spared the risks and costs of unnecessary admission to hospital and more invasive cardiac testing by simply adding a easy to use, immediately obtained, non-invasive test to the diagnostic protocol, and using a threshold of $\mathrm{dP} / \mathrm{dt}_{\mathrm{ejc}}>150$ to rule out heart attack.

\section{DISCUSSION}

The vast majority $(75 \%)$ of patients who visit the emergency department because of chest pain have a normal or nondiagnostic ECG on presentation.

Because patients without ECG evidence of infarction or ischaemia at the time of initial presentation may still have significant coronary artery disease as the aetiology of their chest discomfort, these patients are routinely admitted to hospital to "rule out myocardial infarction" ${ }^{10}$ This admission strategy results in more than three million unnecessary admissions to hospital at a cost of $\$ 10$ billion dollars annually. ${ }^{11}$ One issue driving this liberal admission policy is the concern for "missed" acute MIs. It has been estimated and observed that $2-5 \%$ of all MIs are inadvertently sent home from the emergency department, which proves to be costly in terms of both patient outcomes and legal consequences, ${ }^{12}$ accounting for more than $20 \%$ of malpractice dollars awarded in emergency medicine litigation. In response to this dilemma, there have been many strategies to improve the triage of patients with chest pain and nonischaemic ECG. The goals of these improved strategies are directed to facilitate the timely diagnosis of an occult acute coronary syndrome in the emergency department, while also identifying low risk patients who can be safely discharged home.

At present, ECG and cardiac biomarkers of myocardial necrosis are the conventional tools for detection of ongoing myocardial damage and rule out acute MI at the ER. There are numerous instances in which ECG is not yet diagnostic and the cardiac biomarkers are still within normal limits but myocardial damage is already present. Recently, rest myocardial scintigraphy with tc99m-Sestamibi single photon emission computed tomography (SPECT) (MPI) has been used increasingly in the initial triage of patients presenting with acute chest pain and found to have around $99 \%$ negative predictive value for excluding $\mathrm{MI} .{ }^{10}$ One limitation of rest MPI in the emergency department is that it lacks specificity for acute infarction, because it cannot differentiate between acute infarction, acute ischaemia, and scar from prior MI. ${ }^{13}$

The newly developed non-invasive device described in this article provides a speedy and simple to use alternative method of detecting ischaemia by measuring accurately the ascending limb of the central arterial pressure $\left(\mathrm{dP} / \mathrm{dt}_{\mathrm{ejc}}\right)$ : an accepted index of myocardial performance. ${ }^{14}$ Measurements

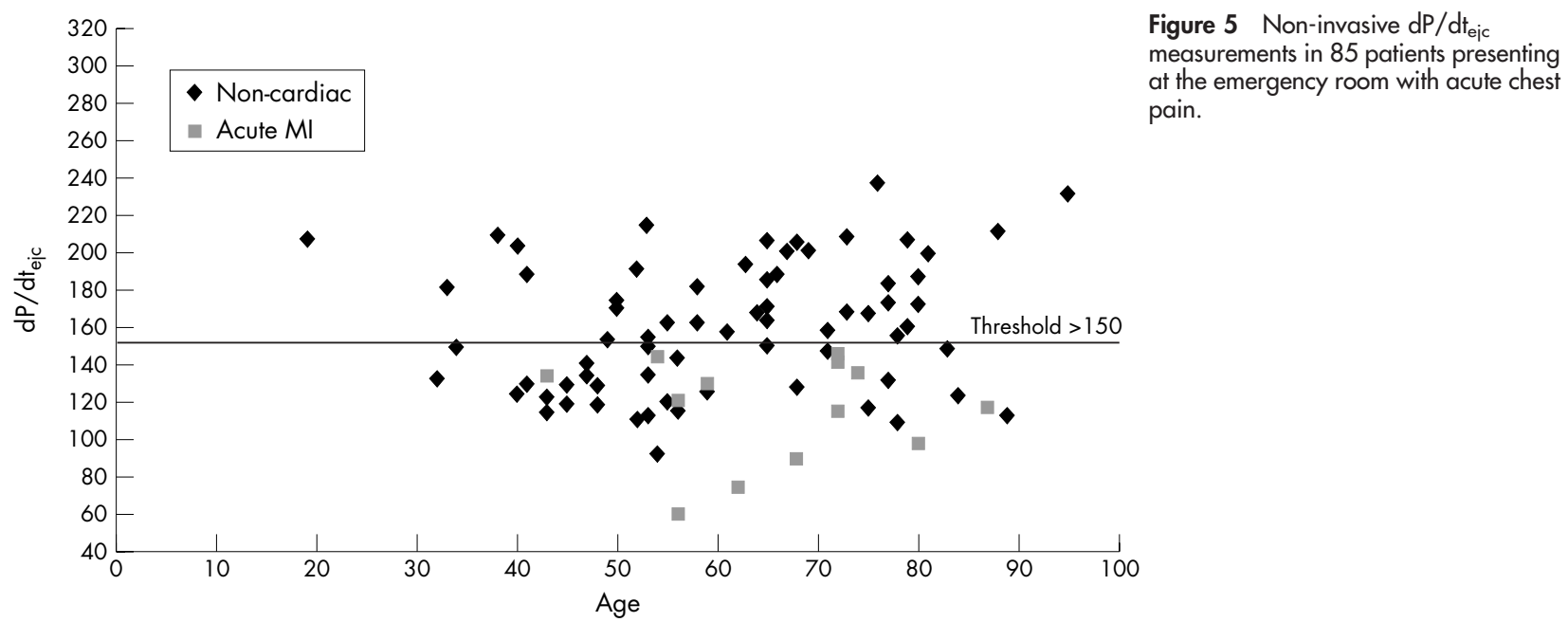

Figure 5 Non-invasive $\mathrm{dP} / \mathrm{dt}_{\mathrm{ej}}$ measurements in 85 patients presenting at the emergency room with acute chest 
take less than 10 minutes and can be performed by a nurse after half an hour of training.

It has been shown that cardiac contractility decreases with a subsequent decrease in left ventricular $\mathrm{dP} / \mathrm{dt}$ and arterial $\mathrm{dP} / \mathrm{dt}$ during acute ischaemia. ${ }^{1-3}$ Mohr et al reported a substantial decrease in arterial $\mathrm{dP} / \mathrm{dt}$ during coronary balloon occlusion. In a previous work, we tested the sensitivity of the new device to detect ischaemic changes during transient coronary occlusion in 16 patients undergoing percutaneous transluminal coronary angioplasty and found that there was a significant decrease in $\mathrm{dP} / \mathrm{dt}_{\mathrm{ejc}}$ immediately after balloon inflation. ${ }^{9}$

Other investigators ${ }^{15}$ measure vasomotor response in the finger using peripheral arterial tonometry by means of a pletismographic device (Itamar Medical, Israel), although it has been demonstrated that in patients with exercise induced ischaemia there is a reduction in pulsatile volume amplitude and local vascular compliance-that is, vasoconstriction. This technique, however, is dependent on the neurovegetative system, which is influenced by many factors, such as local finger temperature changes. ${ }^{16}{ }^{17}$ The limitations of our new method are related to patients with previous MI and severe arrhythmias and patients with severe hypotension; in this subset of patients we may have erroneous results.

\section{CONCLUSIONS}

The current study describes the initial experience with a newly developed device for non-invasive detection of ischaemia. The device measures the arterial rate of pressure rise during ejection $\mathrm{dP} / \mathrm{dt}_{\mathrm{ejc}}$. This haemodynamic parameter is substantially affected by ischaemia and could be used to identify patients, in whom their chest pain is not from cardiac origin, avoiding unnecessary admissions to the hospital and reducing significantly expenses from the health system. Further studies with a larger number of patients are needed to confirm initial findings.

\section{ACKNOWLEDGEMENTS}

We are indebted to Ms Sylvia Walters for assistance during the preparation of this manuscript.

\footnotetext{
Authors' affiliations

M Gorenberg, Department of Nuclear Cardiology and Nuclear

Medicine, Sieff Government Hospital, Safed, Israel

A Marmor, Department of Cardiology, Sieff Government Hospital, Safed, Faculty of Medicine, Technion Institute of Technology, Haifa, Israel
}

H Rotstein, Department of Electrical Engineering, Technion Institute of Technology, Haifa, Israel

Funding: Cardiowatch Ltd, Matam Advanced Technology Center, Haifa, Israel

Competing interests: the authors are stockholders of CardioWatch-a Technion University affiliated company developing the device used in this study.

\section{REFERENCES}

1 Mohr R, Rath S, Meir O, et al. Changes in systemic vascular resistance detected by the arterial resistometer: preliminary report of a new method tested during percutaneous transluminal coronary angioplasty. Circulation 1986;74(4):780-5

2 Mohr R, Meir O, Smolinsky A, et al. A method for continuous on-line monitoring of systemic vascular resistance (COMS) after open heart procedures. J Cardiovasc Surg (Torino) 1987;28:558-65.

3 Mohr R, Dinbar I, Bar-El Y, et al. Correlation between myocardial ischemia and changes in arterial resistance during coronary bypass surgery. J Cardiothorac Vasc Anesth 1992;6(1):33-41.

4 Kantartzis M, Sunderdiek U, Bircks W, et al. Cardiac efficiency during coronary occlusion and during reperfusion after emergency revascularization under cardioprotection. Thorac Cardiovasc Surg 1996;44:20-6.

5 Elhendy A, Schinkel A, Bax JJ, et al. Long-term prognosis after a normal exercise stress Tc-99m sestamibi SPECT study. J Nucl Cardiol 2003; 10(3):261-6.

6 Sharir T, Marmor A, Ting $\mathrm{CH}$, et al. Validation of a method for noninvasive measurement of central arterial pressure. Hypertension 1993;21:74-82.

7 Marmor A, Blondheim D, Gozlan E, et al. Method for noninvasive measurement of central aortic systolic pressure. Clin Cardiol 1987;10:215-21.

8 Marmor A, Sharir T, Schlomo I, et al. Radionuclide ventriculography and central aortic pressure change in noninvasive assessment of myocardial performance. J Nucl Med 1989;30:1657-65.

9 Marmor A, Gorenberg M. A method for early noninvasive detection of myocardial ischemia during abrupt coronary occlusion. Circulation 2003;108(17):541.

10 Abbott B, Jain D. Impact of myocardial perfusion imaging on clinical management and utilization of hospital resources in suspected acute coronary syndromes. Nucl Med Commun 2003;24:1061-9.

11 McCullough PA, Ayad O, O'Neil WW, et al. Costs and outcomes of patients admitted with chest pain and essentially normal electrocardiograms. Clin Cardiol 1998;21:22-6.

12 Freas GC. Medicolegal aspects of acute myocardial infarction. Emerg Med Clin N Am 2001;19:511-21.

13 Udelson J, Beshansky J, Ballin D, et al. Myocardial perfusion imaging for evaluation and triage of patients with suspected acute cardiac ischemia. JAMA 2002;288(21):2693-700.

14 Serruys PW, Wijns W, van den Brand M, et al. Left ventricular performance, regional blood flow, wall motion and lactate metabolism during transluminal angioplasty. Circulation 1984;70:25-36.

15 Rozanski A, Qureshi E, Bauman M, et al. Peripheral arterial responses to treadmill exercise among healthy subjects and atherosclerotic patients. Circulation 2001;103(16):2084-9.

16 Chouraqui P, Schnall RP, Dvir I, et al. Assessment of peripheral artery tonometry in the detection of treadmill exercise-induced myocardial ischemia. J Am Coll Cardiol 2002;40(12):2195-200.

17 Jain $D$, Joska T, Edrisinghe $Y$. Usefulness of peripheral artery tonometry for determining peripheral vascular responses during exercise. Am J Cardiol 2003;91(15):506-10 